\title{
Freeform surfaces adaptation using developable strips and planar quadrilateral facets
}

\author{
Francisco Gonzalez-Quintial*, Javier Barrallo and Ana Artiz-Elkarte \\ Higher Technical School of Architecture, University of Basque Country UPV-EHU, Donostia, Spain
}

\begin{abstract}
Almost all industrialized materials commonly employed at engineering and building construction are approximately unstretchable, metal sheets, plywood or glass. So there is no doubt about the advantages offered, mainly from the economic point of view, by the processes that use flat or developable surfaces in the resolution of doubly curved ones. Using two prototypical kinds of developable surfaces, an adaptation method of double curvature surfaces is formulated using either developable strips or planar quadrilateral surfaces. Through the geometric concept of apparent contours and by the systematization of a process inspired by traditional projective geometry an algorithm is built, using the newest and outstanding CAD processes. They systematize and allow obtaining single-curvature strips or flat facets, in order to be able to address its construction, using materials that can be bent in one direction or rigid material with no possibility of being bent at all by simple and economical procedures. These strips obtained are from the geometrical viewpoint absolutely developable. They are patches extracted from cones or cylinders. The main subject to be developed in this paper is on one hand showing of the system and its geometric basis and on the other the exhibition of the results that are being obtained over physical models and prototypes built in diverse materials.
\end{abstract}

Keywords: Freeform surfaces, double curved surfaces, developable surfaces, algorithmic approach

\section{Introduction}

Architecture in recent years has seen an extensive use of double curvature surfaces. Simultaneously supported in new design and digital fabrication systems there has been an important development in the construction of this kind of surfaces that have allowed to see examples of buildings with spectacular results, like Frank Gehry's designs, supported by the researches and geometric developments of D. Shelden (2002). This has led to a revolution within the systems of geometric control of less conventional shapes, free form and double curvature shapes, not only from the point of view of the CAD technology, but through the impulse that has occurred through the theoretical geometry knowledge and the interesting theoretical developments, as those published by H. Pottmann (2007), intended for specific use in the development of that which is almost a new science, known as Architectural Geometry.

\footnotetext{
*Corresponding author: Francisco Gonzalez-Quintial, Higher Technical School of Architecture, University of Basque Country UPV-EHU. Donostia, Spain. Tel.: +34 943018 407; E-mail: francisco.gonzalez@ehu.eus.
} 
In the works mentioned, a way of approach to geometric resolution of the construction of free form shapes is its adaptation through developable surfaces. Shelden calls them paper surfaces, but Pottmann talks about the generation of planar quad meshes from families of conjugate curves of principal curvature.

This type of planar or developable elements is used because of the simplification and economy that it offers in regard to economic and constructive aspects. A developable surface can be constructed from a planar surface, the other way round a developable surface can be deployed on a plane without any crease or distortion. This question is decisive in manufacturing any element within any constructive system, like a front facade panel or the hull of a ship. From the geometric point of view there are many aspects that define a developable surface. One of them is its gaussian curvature. While a developable surface is characterized by having zero gaussian curvatures, on a free surface any value equal to or different from zero gaussian curvature can be found, whether positive or negative. In this way the adaptation of any freeform surface passes through a process of geometric adaptation, where the degree of restrictions a developable surface must have, is superior to a freeform surface. We can say that a developable surface is undoubtedly less free, by being subject to important geometrical impositions.

In his research $\mathrm{H}$. Pottmann has an amazing new focus and uses mainly a differential geometry basis to elaborate a complete approach to freeform surfaces resolution using developable surfaces, following a refinement process based among others on the use of a principal curve network, which is a special kind of conjugate curve network. This approach allows to obtain both quadrilateral planar facets and developable strips (Pottmann, Schiftner, Bo, Schmiedhofer, Wang, Baldassini, et al., 2008). In the work of Axel Killian (2006) the use of Genetic Algorithms allows an interesting approach to the developable surface issue. The process of adaptation and transformation of a set of strips over a surface supposes a new way to focus on geometric questions. Using an adaptive genoma, an algorithm is built to give a geometric solution through a numerical way. J. Glymph (2004) uses a geometric strategy for generating quadrilateral planar facets. From a constructive point of view this approach is of great interest. This method incorporates basic geometric principles into a parametric framework. So the obtained form is as result of a predefined strategy not an adaptation of any shape.

The aforementioned methods raise and solve similar aims that have been proposed. The method developed is inspired by all of them in some way. However what is proposed hereafter is not an approach based on differential geometry or genetic algorithms. Furthermore reproducing any freeform shape accurately is one of the principal aims of the method. So it is not possible to use a predefined strategy to obtain the form. This is not a form-finding method.

In a similar way as is proposed by Glymph (Glymph, Shelden, Ceccato, Mussel, \& Schober, 2004), the method is based on a classical geometrical basis, mainly on projective geometry expositions as shown by Izquierdo Asensi (1999). Although the method uses a specific kind of conjugate curve network, there is not a differential refinement as in the one Pottman (2007) proposes. It is shown below that apparent contour curves have a conjugate curve network and they have many properties that can be used. Actually these curve networks are quite less regular than principal curve networks but an infinite set of different apparent contour curve networks could be obtained in return. In addition, as will be seen, the use of this specific kind of curves, such as the apparent contour ones, as a basis for the design of developable surfaces, allows to get results that confer a very particular image, and keep up a special link between the perception and the point of view from which the contours are drawn, in other words from which the forms are perceived. 
Furthermore, the use of a generative algorithm, using Grasshopper (Grasshopper, n.d.), has allowed to transliterate the geometric basis as we used to do previously in a projective way. So it has been possible to check and explore the possibilities of these approaches, which could not have been done otherwise.

We have developed the basis of a method of adaptation of double curvature surfaces following the proposed philosophy, trying to respond to both sets of adaptation, as well through the use of developable strips as through planar facets.

\section{Geometric foundations}

When a surface is drawn using any system of representation, whether by hand on paper or by rendering a computer model, it is necessary to define a curve that marks the edge of the represented object. This curve separates the part of the object the observer is able to see, from the part that is hidden. Otherwise it divides the illuminated surface area from the one that remains in the shadow. This is basically the property of the apparent contour curve, also known as separatrix on that surface.

In order to get the apparent contour, an auxiliary projecting surface is used, wrapping the object on the curve that is wanted to be obtained from a defined point of view. This surface is tangent to the object along a series of points that define its apparent contours. It is generated by straight lines or projection rays, starting from the selected point of view they are touching the surface tangentially. These straight lines of projection are the generatrices of a developable surface whether a cone or a cylinder.

The theoretical concept is very simple, however obtaining these apparent contours is complex enough, especially when the object shapes move away from simple primitive shapes. Even on primitive forms, obtaining boundary lines of shadow or separatrices, which is a common application of the apparent contours related to projective geometry, is laborious when it is carried out manually. Using computer-aided design software facilitates and improves this work by providing powerful tools in this regard.

On any surface several apparent contour curves are generated through the displacement of any point of view along any spatial trajectory. Here, straight paths, either proper (Fig. 1) or improper ones, are used (Fig. 2). These apparent contours are generated as tangency between projecting tangent surfaces and basic surface. In this way we wrap the primitive surface with a series of tangent developable surfaces, which are supported on the surface along the apparent contour and intersect each other two by two along an outer curve to the surface.

As a result projecting tangential surfaces are delimited by two curves being external to the primitive surface. These curves are intersections between two by two projecting adjacent surfaces. Besides they contain obviously the proper tangent curve that is the apparent contour. In this way a series of strips are obtained wrapping the original surface externally.

These strips are absolutely developable, since they are obtained from the projecting tangent surface that defines the apparent contour. Its boundaries are determined by two tangent developable surfaces, and the width of the strip in turn by the distance that separates points of projection from which the tangent surfaces are obtained and with them the apparent contours.

The extent of this document does not allow an exhaustive explanation of geometric principles. This process and the details of the geometric basis are profusely detailed in previous works (González Quintial, \& Sánchez Parandiet, 2012). 

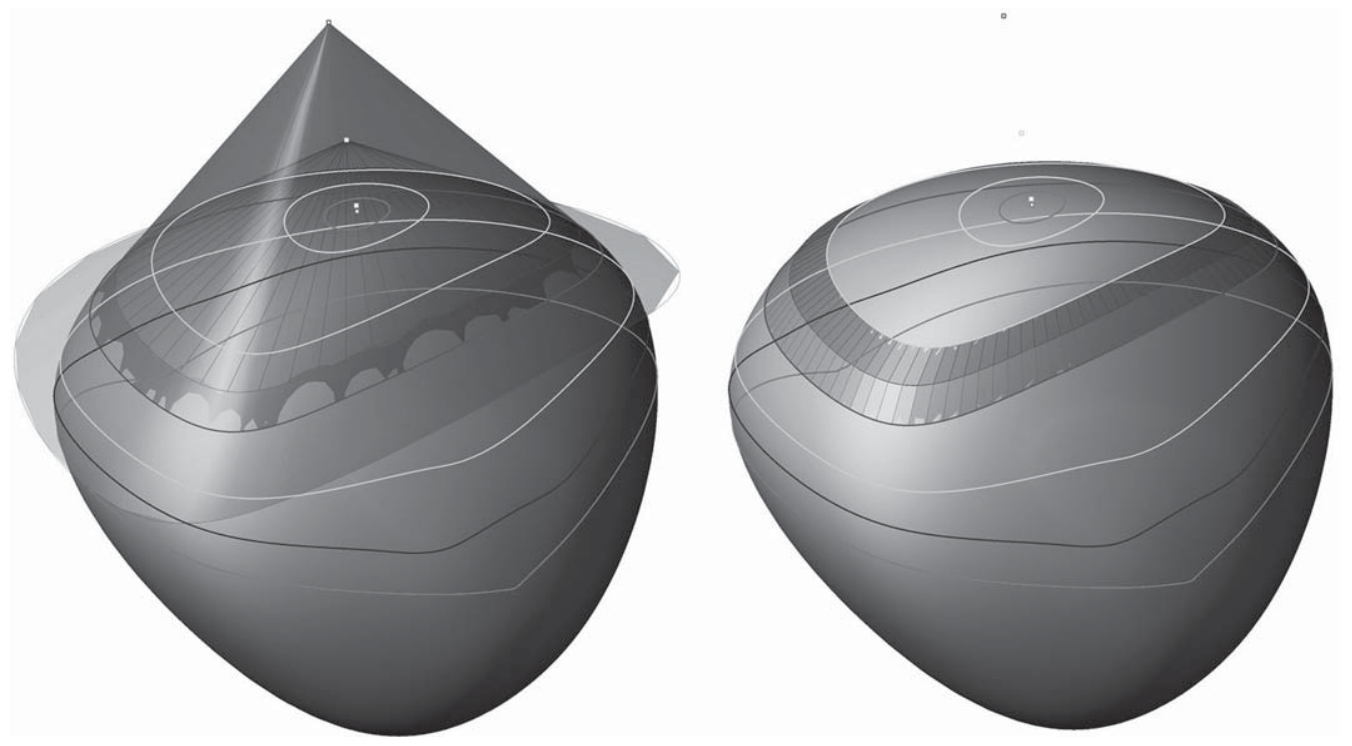

Fig. 1. Contours obtained using proper straight trajectory, projecting conical surfaces.
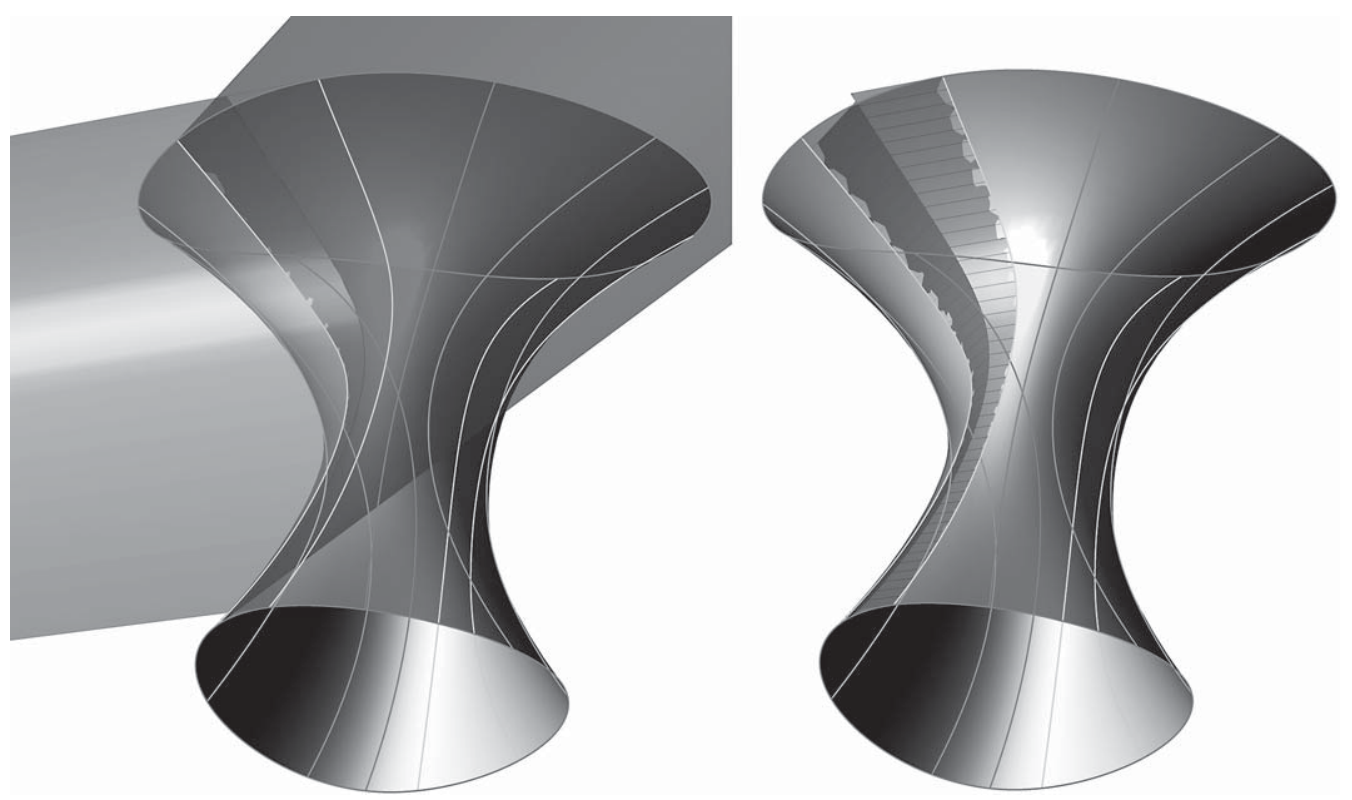

Fig. 2. Contours obtained from improper straight trajectory. Projecting cylindrical surfaces.

\section{Graphical and physical adaptation of double curvature surfaces using developable strips}

This paragraph is about obtaining developable strips that fit freeform surfaces. The problem is the adaptation of this kind of surfaces on which the curvature is not uniform and doesn't have zero 
value. Otherwise, this is the usual situation on complex surfaces or freeform shapes. The success of the strategy focuses on the achievement of an appropriate projection path, which could be satisfactory in terms of setting the apparent contour curves which facilitate ultimately the construction of developable strips.

With the chosen path apparent contours are obtained. It does not depend on any quality of the surface, but it depends particularly on the point of view. There could be an infinite number of apparent contours on a surface, since an object can be observed from an infinite number of positions. The observer, the point of view, is what determines the curve over the surface. The method takes advantage of this flexibility allowing to be related to a mechanism that is subjective, like the human visual perception process. Geometric resolution of the surface has much to do with appearance or the way one is looking.

The process is applied in an empirical way, aided by a system of tracing apparent contour curves, which is built using a generative algorithm in Grasshopper. In this way it is possible to probe multiple options using the same primitive freeform shape; if appropriate, the best set will be chosen, meaning the path that enables to obtain the optimal contour set and consequently allows to ensure a more favorable set of curves which could be used to fit the developable strips.

The adaptation of a freeform surface, where the variation of the curvature is noticeable, is shown in the image (Fig. 3). In this case an improper straight trajectory of projection is used, resulting in apparent contours from tangent surfaces of cylindrical projection. A set of apparent contour outlines should be selected from points of view at the infinite. Here a regular division of the path is defined, along an improper path. From these set of points of view the corresponding set of tangent projecting cylindrical surfaces is generated. The extraction of the patch bounded by the intersections between cylinders allows to obtain a cylindrical developable strip that fits the surface.

Once the graphical way of the surface of double curvature adaption is solved, the checking of the validity of the method must be done by transferring the geometric resolution results to physical models. Several cases have been studied from both graphical and physical points of view through the construction of prototypes using different types of light materials. We are talking about prototypes, not models. On the prototypes and during their implementation process, the behaviour of materials could be anticipated in relation to the way in which they are arranged, and their physical interaction. The prototype has an added value in relation to a purely formal model. The results in terms of tolerances can be checked. Furthermore the deformation and the adaptability in lightweight materials from which the prototypes are made up, anticipate somehow the behaviour of materials that will be used to build the final model.

Consequently, from the constructive point of view, a key feature of the developable surfaces from which the most useful application is obtained, is that theoretically they can be deployed on a plane. As said initially they can be developed without creases or folds, on a flat surface. Conversely, a developable surface can be constructed from a plane. It means that by using any kind of flat or planar constructive element a developable surface can be built.

Because the strips obtained are developable surfaces, it is possible to construct these prototypes using light laminar materials. In other words, it is possible to transfer flat strips unfolding directly, using a cutting machine. As can be seen in the images (Fig. 3 ), a high fidelity degree in the physical reproduction of the model have been achieved. 


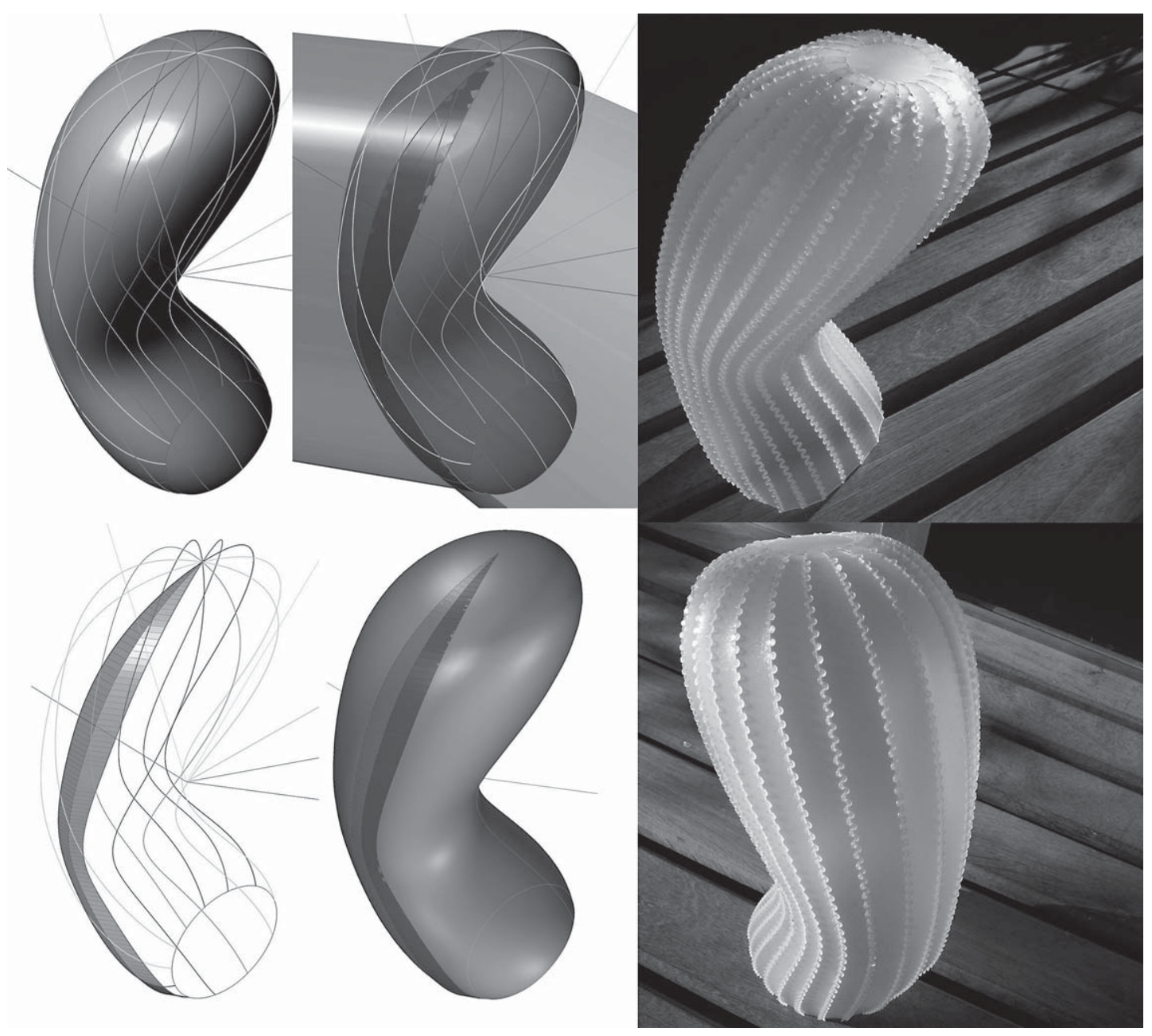

Fig. 3. Development of freeform surface. Application of the method on a freeform surface by a straight improper trajectory. Physical model made applying the process.

\section{Graphical and physical adaptation of double curvature surfaces using planar quadrilateral facets}

The adaptation method of double curvature freeform surfaces through the use of flat panels or quadrilateral facets allows us to generate a mesh, wrapping the surface with flat elements, in order to use this one for the primitive shape construction. This process could be carried out not only using materials that can be curved along one direction, but also using flat rigid materials which do not allow to be curved or bent; these processes imply important extra charges, like glass, otherwise profusely employed, whose process of folding or bending raises its price considerably.

Up to now the apparent contours used to obtain developable strips have been obtained by using points of view located along straight proper or improper lines. The reason is not only a simplification. Of course, using points along straight lines constitutes a better way to order, control and systematize 


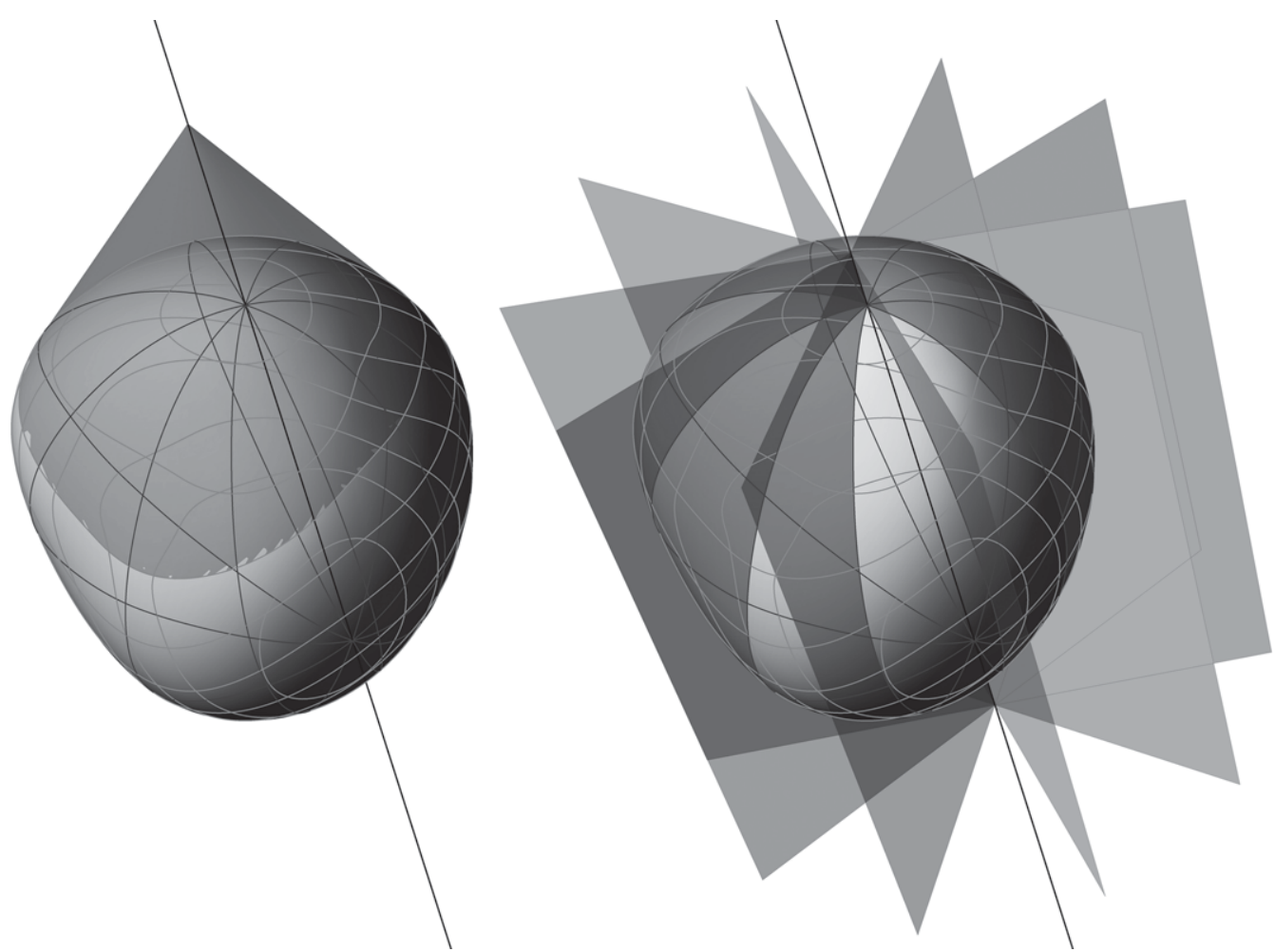

Fig. 4. Generation of apparent contours and conjugated curves using a proper straight path.

the sets of apparent contour curves. It is mainly due to a geometric strategy. This is caused by the fact that if any straight trajectory is used, already being a proper or an improper line, the intersections of the sheaf of planes which are cut along the trajectory and the basic surface (Fig. 4) make up into a group of conjugated curves to the apparent contours set, which were obtained on the original surface using the aforementioned path. They become two families of conjugated curves, thus acquiring all of their properties.

In this way, whatever the conjugated curves networks are, if any curve from the network is taken and the tangent is calculated to the other family curves at each one of the cutting points, these tangents form a developable surface along each of the network curves. Reciprocally this is true as well. Therefore, in our case, by using an apparent contour curve it is possible to calculate their tangents at each of the cutting points with the planar curves of the conjugated curves family. Furthermore, due to their conjugacy property, these ones form a developable surface. In this specific case the tangents are the generatrices of the projection surface.

The projecting surfaces, cone and cylinder, are obviously developable surfaces and both are tangents along the apparent contour curve. In the case of the planar sections, defined by cutting the sheaf of planes and the original surfaces, the result is not as evident, but it can be proved easily. In this way, on one hand it is possible to obtain in an easy way the families of conjugated curves when the paths through which they are traced are straight, and on the other hand it is possible to take advantage of several properties that are very useful in practice. When the path is a proper straight line, the sheaf of planes is easy and directly determinated, using the path as intersection of this sheaf. In the other 

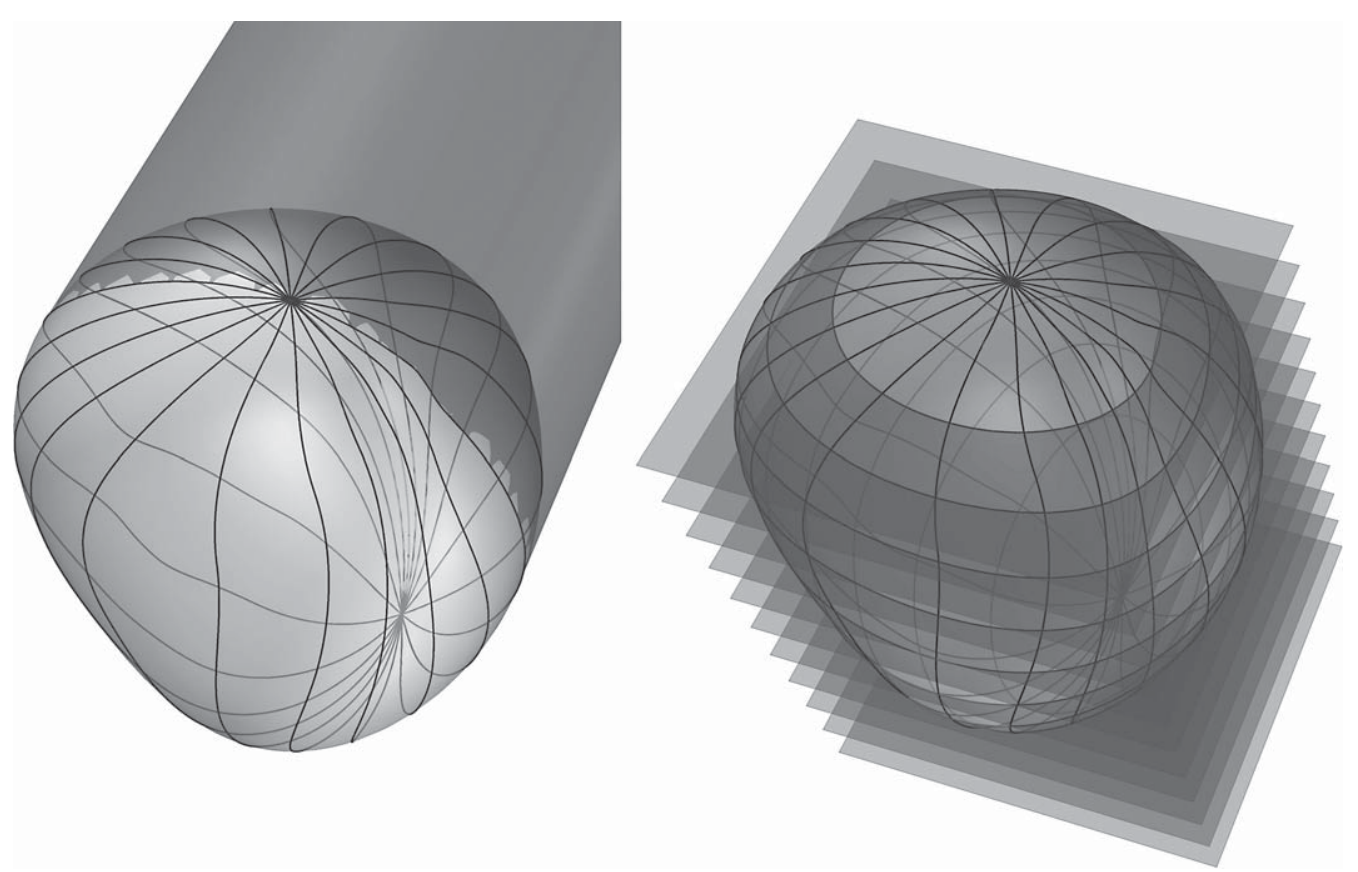

Fig. 5. Generation of apparent contours and conjugated curves using an improper straight path.

case, if an improper line is used, the sheaf of planes cuts at the infinite and a set of parallel planes could be used (Fig. 5).

These properties are profusely used along the method development, due to the fact that from the constructive point of view it is particularly important to have a quadrangular network which could be used on one hand to trace two sets of interwoven strips on the surface and on the other hand, using the same network, it is also possible to get a reticle or mesh whose inner spaces constitute

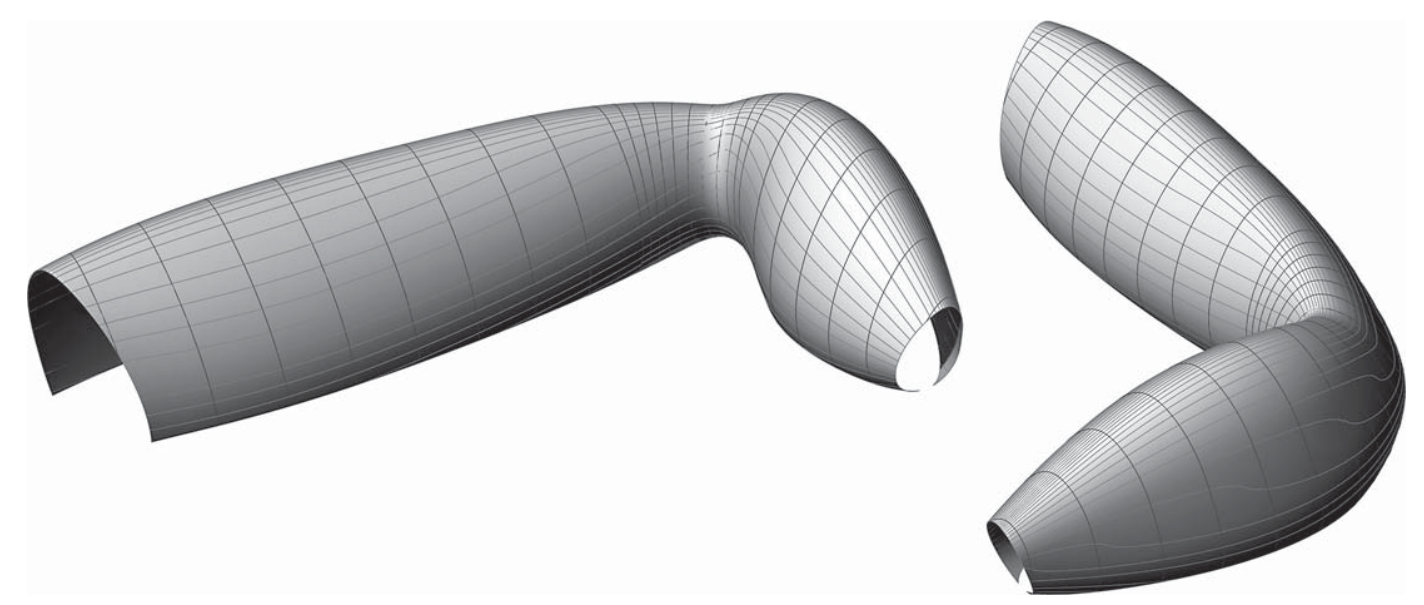

Fig. 6. Conjugated curves families over a freeform surface. In red, apparent contour curves obtained by using several paths; in blue, planar sections under the original surface and the sheaf of planes that contains each trajectory. 

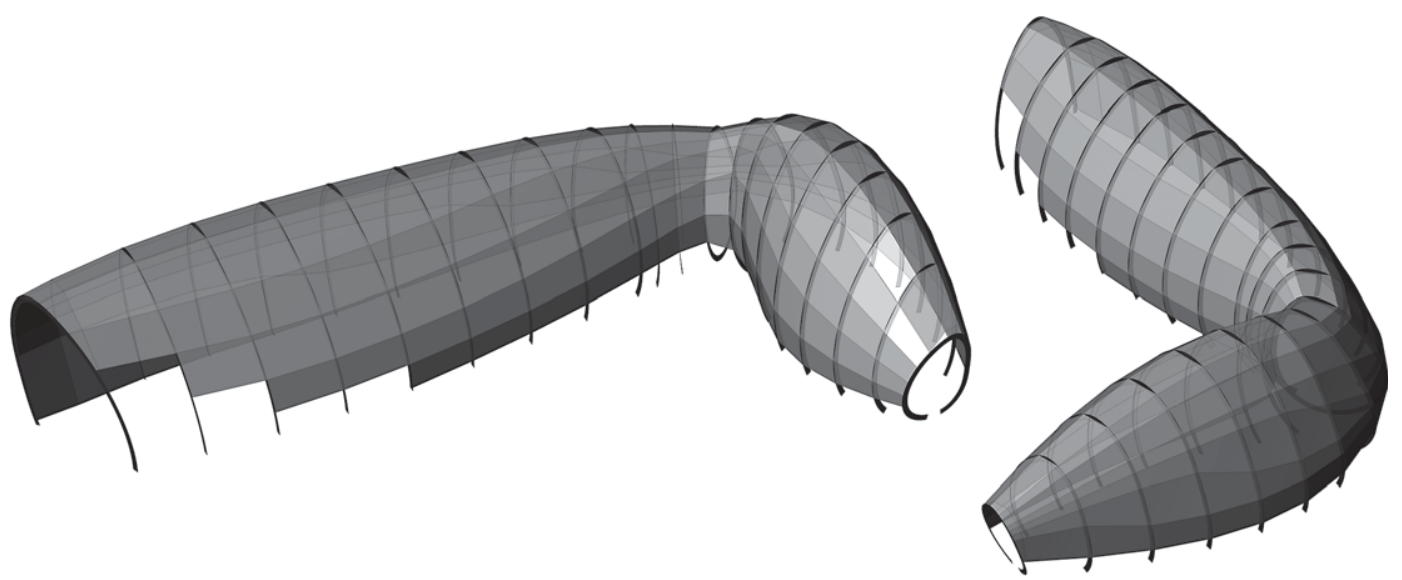

Fig. 7. Planar quadrilateral envelopment derived from the mesh under the conjugated curves network.

planar quadrilateral facets. The less coarse the mesh is, the better approximation to the surface. In this way the general application of the method should be refined on special zones of the surfaces that are adapted (Figs. 6, 7).

In general terms, it is possible to affirm that using a conjugated curves network, obtained from a set of apparent contours, the adaptation of a freeform surface could be carried out through the use of planar rigid materials, like for example laminated glass.
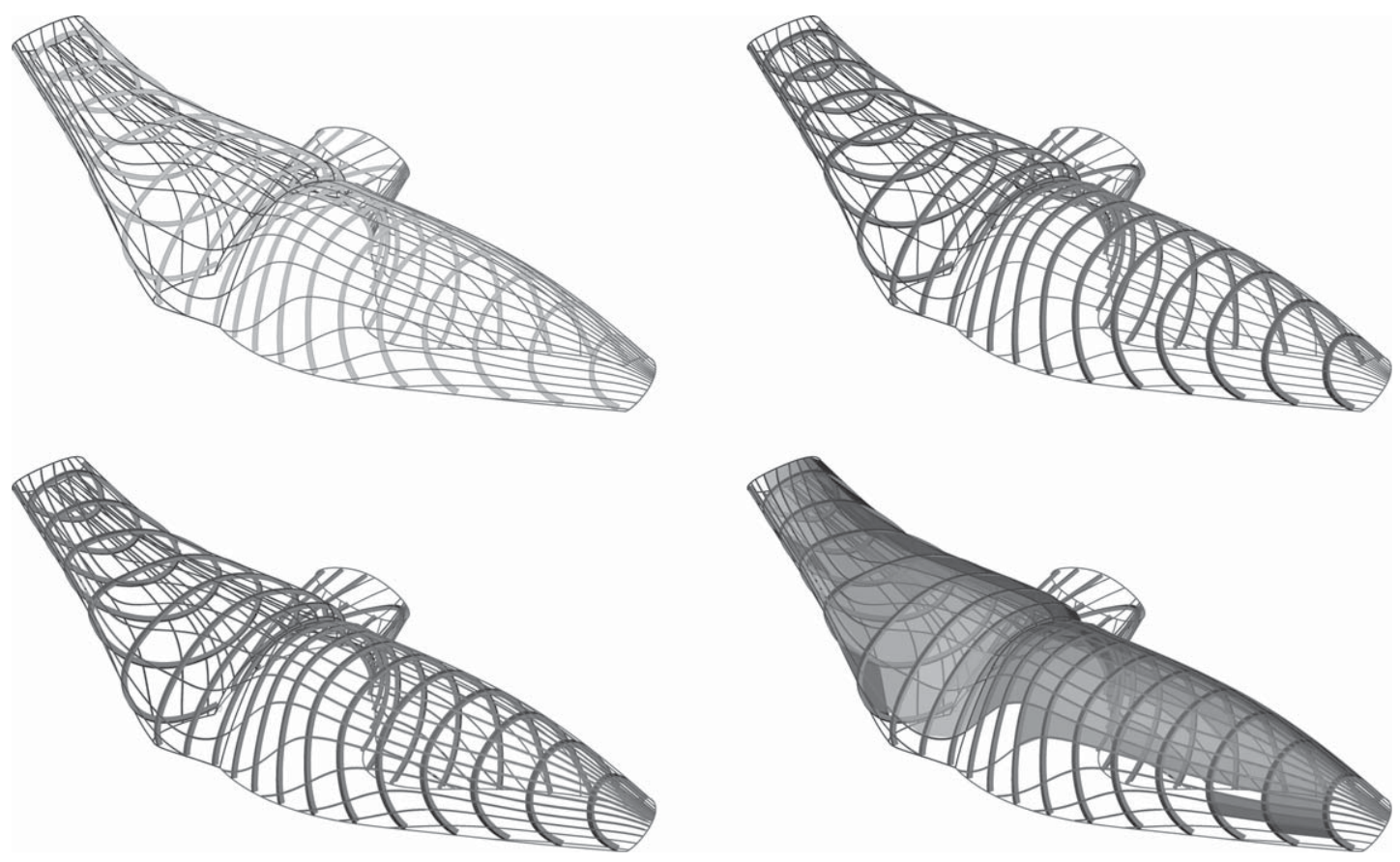

Fig. 8. Graphic resolution of a surface that is not continuous. Contour curves arrangement, planar conjugated sections supporting principal structure and approaching to the envelopments using planar quadrilateral facets. 
Finally, in addition, having a set of planar curves supposes an appropriate starting point of the construction development process of the surface structure and of the approaching to the structural framework. So, the use of planar frames allows for the possibility to utilize structural linear profiles or those that are curved only in their own geometric plane. As a result, from the economic point of view it supposes a much easier fabrication process than methods by which curving is needed of the profiles forming the framework.

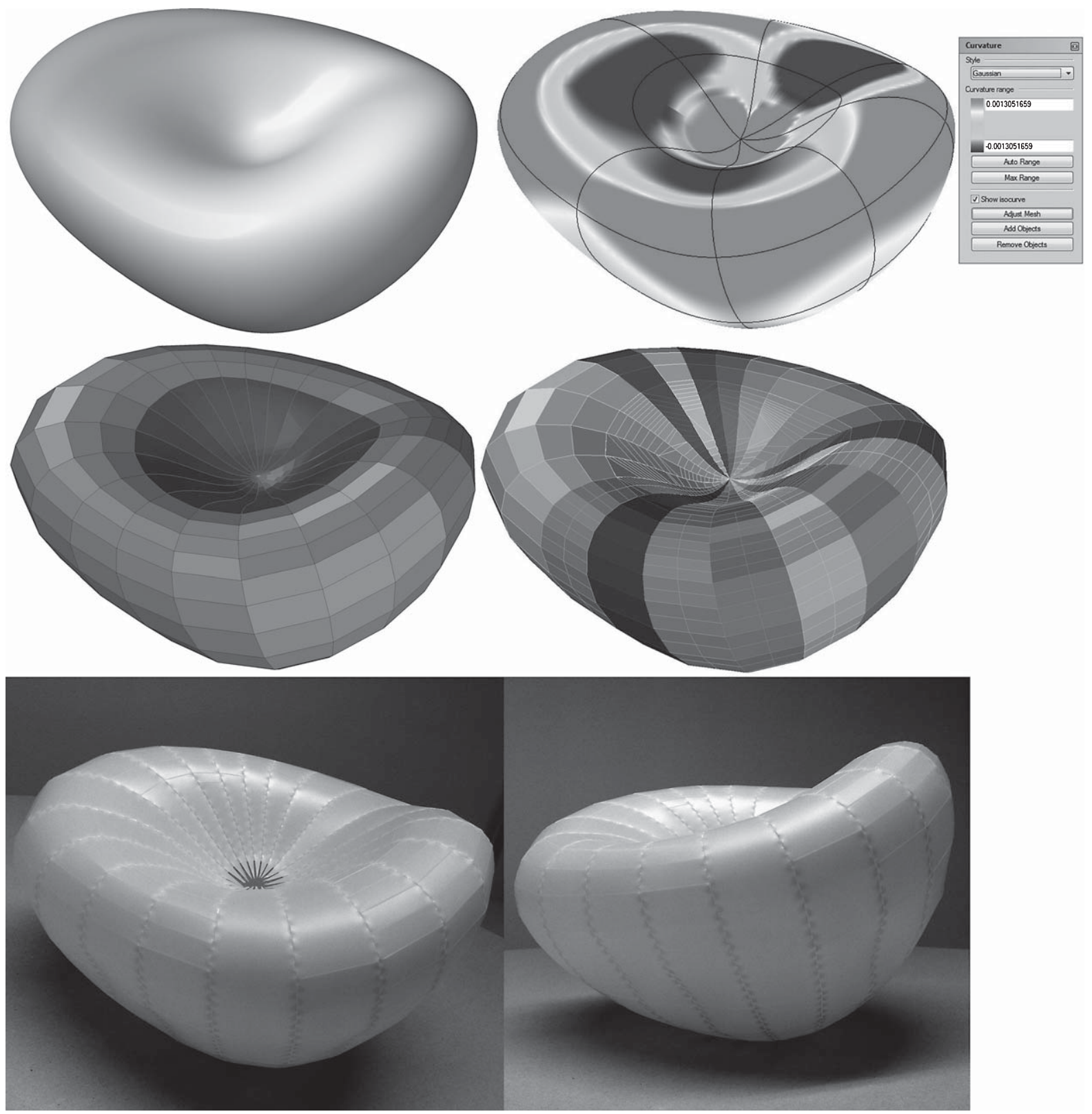

Fig. 9. Adaptation of a variable double curvature surface using complementary strategies articulated through contour lines and their planar conjugated curves. 
In this way as well, both the waterproof envelope as the structural one adapt the geometric shape faithfully. Besides they establish a symbiotic link. So the geometrical approach to the structure and the one used in the construction of the envelop are the same (Fig. 8).

\section{Conclusion}

In conclusion, adaptation is a procedure that implies an alteration of the original shape, so it is necessary to find a balance between constructive economy and fidelity in the reproduction of the form. Based on these premises the theoretical geometric method of adaptation of double curvature surfaces has been formulated, using developable strip surfaces as well as quadrilateral planar facets. The method pretends to be suitable for use over any kind of free form surfaces construction. The formulation of a geometrical method is shown; as we have seen it offers satisfactory results, being suitable for the objectives indicated at the beginning of the paper: graphic adaptation of form and its consequent development and construction, prototypes as well as finished architectural elements.

There are important add-on aspects about the development. The extent of this paper does not allow to show them entirely. First, it is noteworthy to mention the strategy developed to give continuity to the contours on surfaces which are not continuous, i.e. cutting surfaces and tangents, where the contours, due to their own geometric principles, lose their continuity. The flexibility of the layout of contours allows to combine contours, splicing separatrix curves obtained from different points of view, by making a path that chains the contour curves, using their intersections along the secant curve to obtain the tangent planes over the adjoining surface. Each one of the complete contours set obtained over the total surface are not produced using the same point of view, but all points of view are located in the same straight trajectory. The result is a family of contours with a remarkable order and continuity on the set of all the sub-surfaces, as can be seen in Figure 8. Besides, the resolution of the surface in Figure 9 has required some additional strategies consisting of the mix of several theoretical aspects about developable surfaces. In this way it is possible to improve some areas of weakness where the exclusive use of contours do not allow an easy way to build the surface.

The next step in the course of the research is to extrapolate a full-scale construction of these theoretical issues. The method is versatile enough. For example, the construction of the structure and the envelopment of the facade could be considered at the same time, using in that way the same geometric concept. Thus a natural interrelation between both elements is possible. In a certain way these results could have been achieved through the consequences extracted from the prototypes, although it is necessary to check the results in large-scale or one to one scale.

\section{References}

Glymph, J., Shelden, D., Ceccato, C., Mussel, J., \& Schober, H. (2004). A parametric strategy for free-form glass structures using quadrilateral planar facets. Automation In Construction, 13(2), 187-202.

González-Quintial, F. (2012). Método de adaptación de formas de doble curvatura mediante superficies desarrollables. Doctoral Dissertation, UPV-EHU.

González-Quintial, F., \& Sánchez Parandiet, A. (2013). Freeform surfaces adaptation through developable surfaces using apparent contours. Global design and local materialization. Proceedings CAAD Futures (2013), Shanghai, China, 358-367.

Grasshopper (n.d.). Algorithmic modelling for Rhino. Retrieved April 26, 2015, from http://www.grasshopper3d.com/

Izquierdo Asensi, F. (1980). Geometría descriptiva superior y aplicada. Madrid: Dossat. 
70 F. Gonzalez-Quintial et al. / Freeform surfaces adaptation using developable strips and planar quadrilateral facets

Izquierdo Asensi, F. (1995). Geometría descriptiva. Madrid: Paraninfo.

Kilian, A. Embodied Intelligence. Final Project. Using Genetic Algorithms To Generate Developable Strips From Free Formed Surfaces. Overview of Projects. (n.d.). Retrieved April 26, 2015, from http://www.designexplorer.net/

Pottmann, H., \& Bentley, D. (2007). Architectural geometry. Exton, PA: Bentley Institute Press.

Pottmann, H., Schiftner, A., Bo, P., Schmiedhofer, H., Wang, W., Baldassini, N., et al. (2008). Freeform surfaces from single curved panels. ACM Trans Graph, 27(3), 1.

Shelden, D. (2002). Digital surface representation and the constructability of Gehry's architecture. Doctoral dissertation, Massachusetts Institute of Technology, Department of Architecture. 